\title{
Energy Management System and Controlling Methods for a LDH1250HP Diesel Locomotive Based on Supercapacitors
}

\author{
Marius Cătălin Carp, Ana Maria Puşcaş, and Paul Nicolae Borza \\ Transilvania University of Braşov, Electrical Engineering and Computer Science \\ 29 Eroilor Street, 500036, Braşov, Romania \\ \{ana_maria.puscas,marius.carp\}@yahoo.com, paul.borza@unitbv.ro
}

\begin{abstract}
The present research is focused on developing solutions for increasing the lifetime of the batteries used on diesel locomotive. In order to increase the lifetime of the batteries and to reduce the fuel consumption and the pollutants, an intelligent starting system of the diesel locomotive is proposed. The starting system is composed of supercapacitors, batteries and adequate control system used for controlling the power flow from the storage devices to the internal combustion engine in the starting process. The implementation is described, modeled and simulated and the results of the simulations are compared with the experimental ones.
\end{abstract}

Keywords: supercapacitors, embedded control, energy management, diesel locomotive, lifetime.

\section{Introduction}

Lately, the population became more and more dependent on transportation. Even if we speak about public transportation (buses, metros, railways) or personal vehicles, the trend of the fuel and energy consumption is alarming increasing.

Developing new solutions, technologies and methods for improving the energy efficiency represents a priority line in order to satisfy the actual energy requirements.

In transportation, to improve the performances of the storage devices researches are made. The new researches from nanotechnology field allowed developing new electric storage devices with increased performances, such as stacked supercapacitors (SSC) [1], [2], [3]. The transportation field had as target implementing different systems based on SSC that allow the displacement of the vehicles using the self energetic resources. Among such systems, it can be mentioned: (i) the subway from Moskow where an emergency system based on the energy stored on SSC and batteries allow the displacement of the metro train in emergency cases to the first station [4], (ii) the hybrid bus developed by NASA [5], (iii) diesel locomotives developed by institutes such as ELTechnology and "Werkstoffe \& Technologien, Transfer \& Consulting" [6], [7]. Even if there are multiple implementations related to this topic, this research still represents a thematic of scientific interest, the focus being oriented on increasing the lifetime of the batteries used in the staring process. Also, the research is focused on 
offering major improvements of the power trains of the self drives structures thus increasing their energy efficiency. In present, the starting systems of the ICE based on a combination of batteries and SSC represents a major applications class.

The aim of this research is to improve the energy efficiency, the reliability and the performances of the actual starting systems implemented on the self drive mobile structures that use internal combustion engine (ICE). The goal of the research is to modify the architecture of the starting process of a classic LDH1250HP diesel locomotive (LDH) in order to increase the lifetime of the batteries, the energy efficiency and to reduce the fuel consumption and pollution. Thus, the present paper presents the design, the electric model, the prototype and the experimental results of the implemented system used for starting the LDH diesel locomotive.

\section{Contribution to Sustainability}

The present research significantly improves the actual starting systems of the ICE based on a combination of batteries of accumulators and SSC. The advantages of the prototype are related to reducing the number of the accumulators thus reducing the carried weight. By using combined solutions for storage devices (SSC - non polluting storage devices) the number of the starts and stops can be increased without having the performances of the batteries affected, thus reducing the carbon footprint. Thus, the running time of the $\mathrm{LDH}$, the pollutant emissions and the fuel consumption can be significantly reduced. Also, by using the implemented starting system controlled by a microcontroller scheduler, the lifetime and the performances of the batteries and also the reliability of the ensemble are significantly improved. This prototype creates new opportunities in the field of power electronics used in the heavy transportation systems, increases the energy efficiency, increase the exploitation efficiency thus being in a good agreement with the principles of the sustainability.

\section{Architecture of the System}

In order to asses the goal of the present research, the architecture of the LDH was modified. The classic structure of the LDH is supplied from two banks of lead acid accumulators connected in parallel. One bank of accumulators consists in 8 $12 \mathrm{~V} / 160 \mathrm{Ah}$ lead acid accumulators connected in series. In the new structure of the $\mathrm{LDH}$, one of the two banks of accumulators (the second supplying branch) was replaced with three $12 \mathrm{~F} / 110 \mathrm{~V} \mathrm{SSC}$ connected in parallel, able to provide the equivalent power density of $218 \mathrm{~kJ}$ [8], [9], [10]. As consequence, the size of the replaced branch was reduced to half of its initial size and the power density was increased. Thus, the researched starting system is composed of lead acid batteries with high energy density and SSC with high power density for providing the high peak current pulses requested in the starting process, even when the ambient temperature is bellow zero centigrade thus increasing the lifetime of the battery.

\section{Physical Implementation}

The architecture of the prototype is illustrated in Fig. 1, the starting system in Fig. 2 and the control algorithm implemented on ECS and used for the starting system in 
Fig. 3. The charging system includes current limitation and voltage regulator devices able to protect the batteries after the ICE is stated. By switching on K3, the SSC is also charged at a constant current.

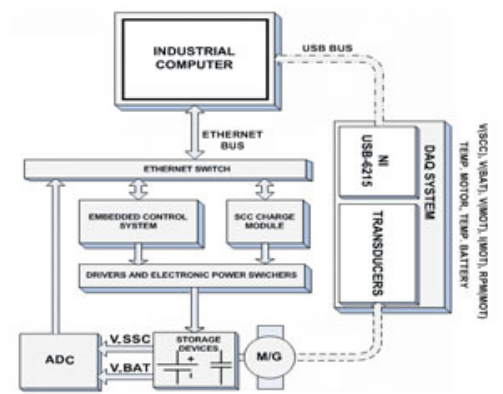

Fig. 1. General architecture of the prototype

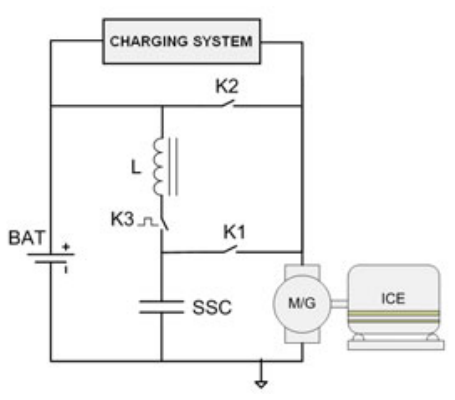

Fig. 2. Implemented starting system

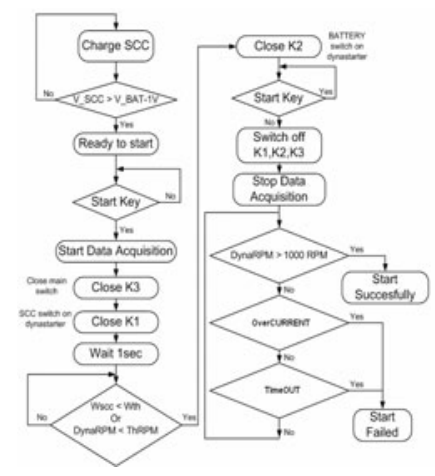

Fig. 3. Block diagram for starting the ICE of the LDH

The implemented and tested prototype consists in: (i) industrial computer, (ii) National Instruments data acquisition system (NI-DAQ), (iii) Ethernet network, (iv) electric high power devices (SCR) and (v) embedded control system (ECS) used for ensuring the correct power flow in the starting system.

As it can be seen in Fig. 3, before any start is performed, the voltage level of the SSC is automatically verified. If its voltage is below $96 \mathrm{~V}$, the starting process will begin by charging the SSC from the battery at a limited current (K3 - switched on), thus protecting the battery. A flag indicates when the LDH can be started by the operator. After the starting button is pressed, the switches are firstly commuted on supplying the LDH from the SSC and after its energy is consumed, the starting process automatically commutes on battery in order to ensure the energy for maintaining the starting process. After the ICE is running, the switches are commuted thus ensuring the charging process of the SSC from the ICE. A voltage regulator was used in order to charge and to limit the voltage on the SSC and battery pack at $110 \mathrm{~V}$, thus avoiding 
the overcharging process which can damage the storage devices. If the velocity of the ICE does not reach its nominal value (1000 RPM) or the current absorbed from the battery is greater than a threshold current of $600 \mathrm{~A}$, the fault situations can appear and an error is signalized. In the normal operation stage, the SSC play the role of a capacitive filter for smoothing the voltage on the electric circuits of the LDH. More than that, in case of switching off the locomotive, the SSC will maintain the maximum voltage level (around $96 \mathrm{~V}-110 \mathrm{~V}$ ) for several hours. Thus, the voltage level on the SSC will facilitate the next staring process, the recharging of the SSC not being necessary anymore [2], [11], [12].

\section{Simulations vs. Experiments and Results}

Before implementing the system, to observe the behavior of the starting process of the LDH, simulations were made in PSpice tool. The model of the starting system is illustrated in Fig. 4 and the results of the simulations are illustrated in Fig. 5.

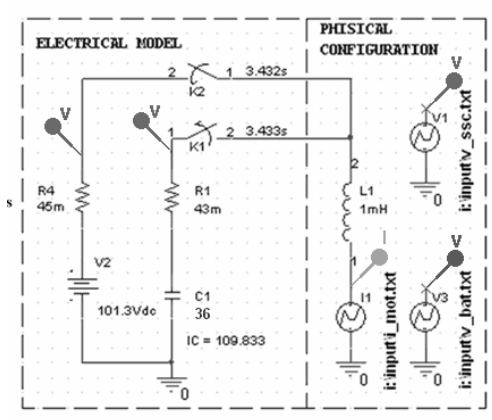

Fig. 4. Model of the starting system

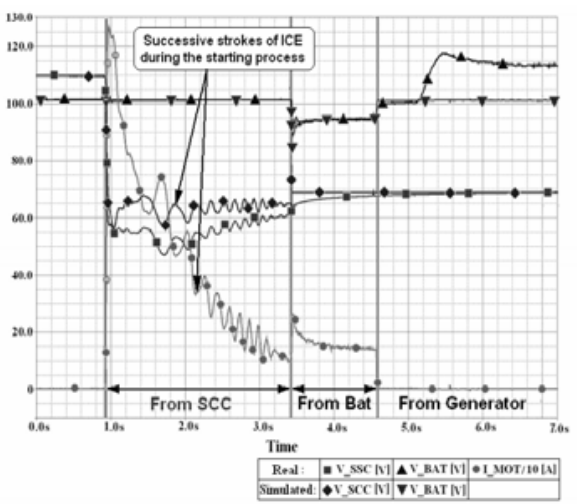

Fig. 5. Comparison between real and simulated experiments of the LDH starting system - battery and supercapacitor voltage and current

As it can be seen from Fig. 4, the model of the starting process of the LDH is composed of the 2 branches of storage devices (pack of batteries and pack of SSC) connected to the ICE through 2 switches (K1 and K2) electronically controlled. For modeling the packs of SSC and batteries, ideal models were used. The results of the simulations are compared with the real values of the current absorbed by the ICE in the staring phase.

As it can be seen in Fig. 5, the results of the simulations follow the experimental results. The current requested by the starting process of the LDH in the first $2.5 \mathrm{sec}-$ onds is provided by the pack of SSC. A centrifugal mechanical regulator for controlling and maintaining the revolution speed at the desired value is used by the ICE. The centrifugal mechanical regulator automatically disconnects the ICE if the revolution is not maintained at its nominal rate for about 2 seconds after the starting was made. In order to ensure these requirements, the ICE is electronically switched to be supplied 
from the batteries. As it can be seen from the experiments and simulations, the current provided by the battery in the starting process is about 5 times smaller than the current provided by the SSC (Ibat $=16 \% * I s c)$. Thus, the implemented system is protecting the battery from the high peak current pulses, they being provided by the SSC.

The difference in the behavior of the starting process between the simulations and results is due to the ideal models from PSpice used in the simulation process. To increase the accuracy of the simulation, complex models have to be used.

In Fig. 6 the calculated power and energy flow provided by the storage devices (SSC and batteries) to the load (ICE) can be observed. These values are determined by using the raw data -voltage and current - recorded with NI-DAQ. The ability of the SSC to provide peak power relative to the batteries can be observed. As result, in Fig. 6 the slope of the energy provided by the $\operatorname{SSC}(\alpha)$ is grater than the slope of the energy provided by the pack of batteries $(\beta)$.

In order to take the right conclusions, a data acquisition system mounted on the $\mathrm{LDH}$, necessary to acquire the signals directly from the SSC, batteries and ICE was developed. Thus, an on - line software used for data acquiring and monitoring was implemented by using Visual Studio .NET tool.

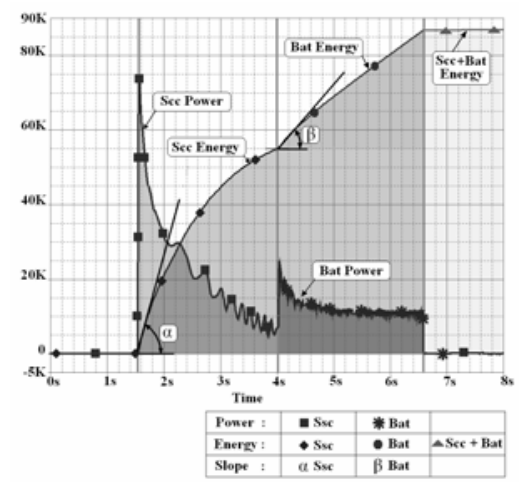

Fig. 6. Calculated power and energy corresponding to LDH starting process

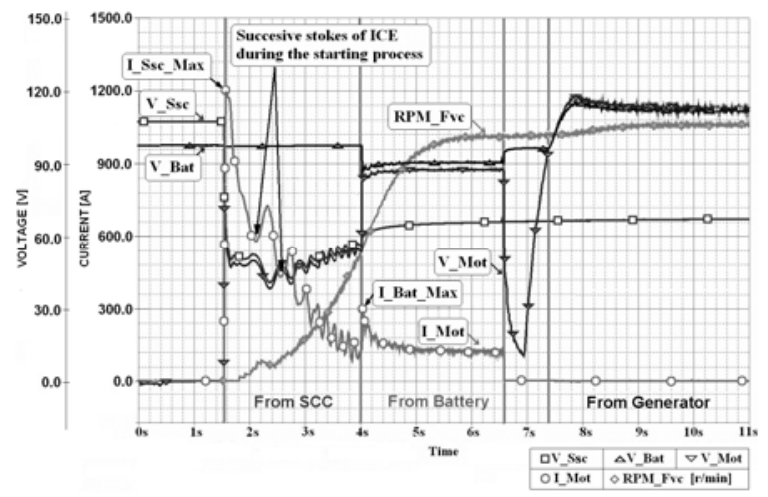

Fig. 7. Real records of the main parameters that characterize the LDH starting process 


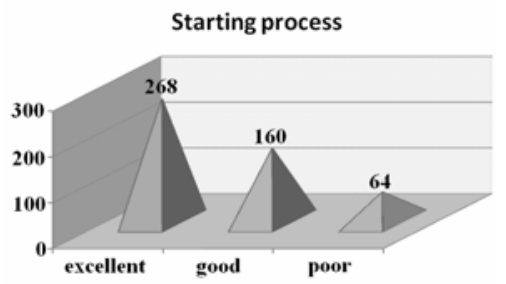

Fig. 8. Statistic of the starting processes of the LDH ICE

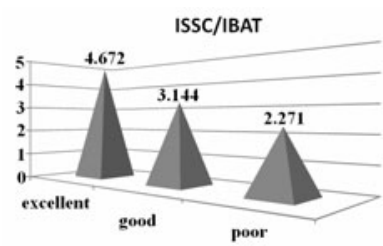

Fig. 9. ISsc/IBatt ratio

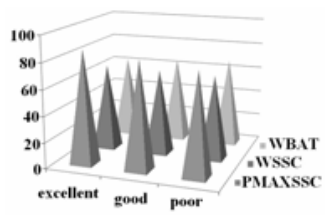

Fig. 10. PMaxSsc, WSsc, WBatt variation

The time variation of the voltages and currents - on SSC and batteries and also the revolution speed of ICE during a real staring process is illustrated in figure Fig. 7.

After defining several qualitative factors of the starting system, based on the acquired data, a statistic was realized. The chosen factors are: (i) the rate between the maximum current provided by SSC at the stating time and the maximum current supplied by the batteries at their commutation into the DC starting motor circuit (I_Ssc_Max/I_Bat_Max), (ii) the voltage variation rate of the batteries at their commutation time on the DC starting motor, (iii) the maximum power provided by SSC and batteries during the starting process, (iv) the energy provided by the SSC and batteries during the starting process and (v) the revolution speed. All these data illustrate the accuracy of the starting process of the locomotive. We have also taken into account the temperature of the ICE and the ambient temperature. The real records reveal a normal variation of the above mentioned parameters. These data have been statistically analyzed and classified by labeling the starting processes as poor, good and excellent. The variation of the voltage on SSC at the initial moment was ignored because two situations were identified: (i) charging the SSC from the batteries as a result of a long resting period, (ii) charging the SSC from the generator of the LDH when the time period between the charging process of the SSC and the starting process of the LDH was short.

The statistic results are illustrated in Fig. 8, Fig. 9 and Fig. 10. From the total number of the starting process, only $1 \%$ was wrong and ignored. The successful rates of the starting process are illustrated in Fig. 8. From the total starting processes only $13 \%$ were classified as being "poor", and $87 \%$ as being good or excellent. These statistic results validate our implemented and tested prototype.

The qualitative parameters mean values for the classified records are illustrated in Fig. 9 and Fig. 10. As it can be seen in Fig. 9, a good rate of the ISSC/IBAT ratio is 
around $500 \%$. Thus, because of the significant reduction of the current provided by the batteries its reliability means a high protection of the batteries during the starting process of the LDH.

In Fig. 10 is illustrated the variation of the power and energy on SSC and batteries. The first series prove the dependency of the quality of the starting process to the power level injected by SSC during the first $2.5 \mathrm{~s}$. The amount of the energy transmitted to the ICE is almost the same in all cases, thus emphasizing the importance of the power time dependencies offered by our system during the starting process.

\section{Conclusions and Future Work}

Usually, the performances and the lifetime of the batteries are affected especially by the high peak current pulses required by the ICE in the starting process. Because the implemented system is able to provide these peak current pulses from the SSC, the number of starts and stops of the LDH can be increased without the performances of the battery being affected. Thus, the size of the batteries was reduced to half of its initial value and the daily fuel consumption of the LDH used into the depot was reduced with around 6\%. As consequence, the pollutants were also reduced. By using for the majority of the starting processes pre-charged SSC in previous displacements, the batteries are substantially protected, the biggest part of the power being supplied from the SSC.

The present paper describes the architecture of the prototype endowed with protection and self adapting features. Also, the algorithm detailed in the paper was used for controlling the correct functionality of the system and also contributed at increasing the performances of the starting system.

The paper also introduces a model of the starting process which was validated by the experimental results.

By using the experimental data acquired in 6 months (more than 1300 records), a statistic was made, thus determining the successful rate of the starting process. The results validated the prototype.

A set of criteria that reveal the accuracy of the starting process was defined and used for analyzing and classifying the experimental results. These criteria were applied for a collection of 492 samples from the total number of 1300 records. The simulations and experimental results clearly demonstrate the efficiency of the implemented system in the process of increasing the lifetime of the battery.

As future work, by identifying the real parameters of the system, a software sizing tool will be conceived. This toll is necessary to minimize the size of the batteries and supercapacitors by correlated these with the characteristics of the ICE.

Acknowledgments. This paper is supported by the Sectoral Operational Programme Human Resources Development (SOP HRD), financed from the European Social Fund and by the Romanian Government under the contract number POSDRU/6/1.5/S/6. Also, the present paper is a part of the national "TRANS-SUPERCAP" D21018/2007 project, currently under development at Transilvania University of Brasov. 


\section{References}

1. Conway, B.E.: Electrochemical supercapacitors - scientific fundamentals and technological applications. Kluwer Academic/Plenum Publishers, New York (1999)

2. Chesa, A.: Locomotiva diesel hidraulica de 1250CP, pp. 382-390. ASAB Publisher (2001)

3. Sojref, D., Kuehne, R.: Supercapacitors - the European Experience in Transit Applications. In: Advanced Capacitor World Summit 2008, San Diego, CA (July 2008)

4. Sukhorukov, A.I.: Movement of metro train by supercapacitor - Electric train repair plant of Moscow metro. COST Action 542, Presentation

5. http://ntrs.nasa.gov/archive/nasa/casi.ntrs.nasa.gov/1998001 3899_1998058116.pdf

6. http://www. cantecsystems.com/ccrdocs/ELIT-TechnologyOverview.pdf

7. http://www.trecstep.com/German_Startup_Diesel_Start_engl.pdf

8. Petreus, D., et al.: Modeling and Sizing of Supercapacitors. Advances in Electrical and Computer Engineering 8(2), 15-22 (2008)

9. Diab, Y., et al.: Self Discharge Characterization and Modeling of Electrochemical Capacitor Used for Power Electronics applications. IEEE Transactions on Power Electronics 24(2), 510-517 (2009)

10. Bohlen, O., et al.: Ageing behavior of electrochemical double layer capacitors Part I. Experimental study and ageing model. Journal of Power Sources 172, 468-475 (2007)

11. Carp, M.C., et al.: Monitoring system and intelligent control system used in the starting process of a LDH1250HP. In: 12th OPTIM 2010, pp. 551-556 (2010)

12. Sojref, D., Borza, P.: Comparison of High-Voltage Supercapacitor Approaches and Case Study in Diesel Locomotive Starting System. In: 3rd European Symposium on Supercapacitors and Applications: ESSCAP 2008 (2008) 